\title{
Investigating the role of macrophages in tumor formation using a MaFIA mouse model
}

\author{
A.B. CLIFFORD ${ }^{1}$, A.M. ELNAGGAR ${ }^{1,2}$, R.A. ROBISON ${ }^{1}$ and K. O'NEILL ${ }^{1}$ \\ ${ }^{1}$ Department of Microbiology and Molecular Biology, Brigham Young University, \\ Provo, UT, USA; ${ }^{2}$ Ain Shams University, Cairo, Egypt
}

Received April 17, 2013; Accepted May 15, 2013

DOI: $10.3892 /$ or.2013.2508

\begin{abstract}
Tumor-associated macrophages (TAMs) interact with tumors in their development, growth and metastatic activities. Using a transgenic mouse model that allows for the selective depletion of macrophages we were able to access the macrophage's potential to facilitate metastasis. In the MaFIA (Macrophage Fas-Induced Apoptosis) mouse, transgeneexpressing cells of the myeloid lineage undergo death by apoptosis in the presence of the drug AP20187. Enhanced green fluorescent protein (EGFP) was fused to the suicide gene to allow identification of transgene-expressing cells. Tumor induction was accomplished by subdermal and intravenous injections of B16-F10 melanoma cells. Metastasis in mice with depleted macrophages was compared to metastasis in normal control mice. The lungs and kidneys were examined for metastatic cells. The macrophage-depleted groups showed significantly less metastasis $(\mathrm{P}>0.001)$ compared to the control groups. We theorize that macrophages may aid the metastatic process by fusing with melanoma cells. Using appropriate cell markers and fluorescence-activated cell sorting, we were able to detect a small population of double-positive cells. We confirmed cell fusion by microscopic analysis, visualizing the cell's morphology by both immunohistochemistry and immunofluorescence. The presence of double-positive cells suggests macrophage/cancer cell fusion could be a possible mechanism for metastasis.
\end{abstract}

\section{Introduction}

Macrophages perform a variety of functions from phagocytosis and the release of pro-inflammatory cytokines, to the repair and remodeling of damaged tissues. Two phenotypes have been proposed to identify their diverse roles: types M1 and M2 (1). The M1 macrophage refers to the cell which aggressively

Correspondence to: Professor K. O'Neill, Department of Microbiology and Molecular Biology, Brigham Young University, 855 WIDB, Provo, UT 84602, USA

E-mail: kim_oneill@byu.edu

Key words: MaFIA mice, macrophage depletion, tumor metastasis destroys foreign invaders and tumor cells, and releases proinflammatory cytokines. The M2 macrophage acts as a support cell by promoting the healing of damaged cells by angiogenesis and tissue remodeling. The transition from M1 to M2 might be facilitated by tumor environments (1). Tumors produce vascular endothelial growth factor (VEGF) and macrophage colony stimulating factor (M-CSF), both of which have the ability to interact with tyrosine kinase receptors and induce macrophage migration $(2,3)$. The hypoxic center of the tumor releases factors that attract an M1 type, but as the macrophage enters the tumor microenvironment, it is exposed to a variety of cytokines. These cytokines may possibly convert type M1 macrophages to M2. In this microenvironment, Interleukin-10, (IL-10), IL-6, IL-4, transforming growth factor $\beta$ (TGF- $\beta 1$ ), and prostaglandin F2 (PGF2) are present. These factors inhibit the cytotoxic activity of macrophages (4). In addition; IL-10, IL-4 and IL-13 do more than just inhibit macrophage activation. IL-10 induces a specific activation program and leads to alternatively activated macrophages. This exposure to IL-10, and possibly IL-4 and IL-13 has been suggested to convert M1 macrophages to an M2 phenotype (1). The possibility that aggressive macrophages (M1), programmed to destroy cancerous cells, might become converted to ones (M2) whose functions aid and promote tumor survival is alarming, and would be a promising target for cancer therapy.

Tumor-associated macrophages (TAMs) are thought to behave and function as M2 macrophages in their abilities to aid tumor cell survival and growth. In certain cancers, such as breast cancer, TAMs can comprise as much as $50 \%$ of the tumor mass (5). High numbers of TAM infiltrates in tumor masses correlate with poor prognosis in breast, prostate, ovarian, cervical and lung cancer (6). This poor prognosis could be due to a variety of cytokines expressed by TAMs that stimulate tumor cell proliferation and survival. TAMs have been shown to release a number of pro-angiogenic cytokines and growth factors, including, epidermal growth factor (EGF), platelet-derived growth factor (PDGF), TGF- $\beta 1$, hepatocyte growth factor (HGF), basic fibroblast growth factor (BFGF), vascular endothelial growth factor (VEGF), tumor necrosis factor $\alpha$ (TNF- $\alpha$ ), and IL-8 (6). TAMs also contain various angiogenesis modulating enzymes, such as matrix metalloproteinases (MMP) MMP-2, MMP-9, MMP-7, MMP-12 and cyclooxygenase 2 (COX-2) (4). The many pro-angiogenic functions of TAMs explain the correlation found between 
increased TAM numbers and high vascular grades of many tumor types (7). By stimulating the growth and proliferation of tumor cells, TAMs have been shown to promote tumor development. An important question still remains, could TAMs help in metastatic progression as well?

Studies of the migration of mammary carcinoma cells in primary tumors indicated the migration to be regulated by macrophages (8). In vitro fusion of weakly metastatic mouse Cloudman S91 (6neo) melanoma cells with peritoneal macrophages from DBA/2J mice produced hybrids, a majority of which displayed enhanced metastatic potential in vivo (9). It has also been shown that co-culturing tumor cells with macrophages increases the tumor cell's invasive properties (10). Further studies have indicated that TAMs are associated with tumor cells that move away from the main body of the tumor (11). The movement of tumor cells into blood vessels frequently occurs at sites containing clusters of macrophages (12). All of these observations indicate a metastatic association between TAMs and cancerous cells. According to Pollard, macrophages are educated by the tumor microenvironment, so that they adopt a trophic role that facilitates angiogenesis, matrix breakdown and tumor-cell motility: all of which are elements of the metastatic process (13).

To further investigate the possible relationship between TAMs and metastasis, we used the Macrophage Fas-Induced Apoptosis (MaFIA) mouse. In the transgenic MaFIA mouse, the cells of the myeloid lineage have a transgenic receptor that when exposed to the drug AP20187 (Ariad Pharmaceuticals), induces selective cell death by apoptosis. These mice have been developed with a special cellular receptor gene under the control of a macrophage specific promoter, the $c f m s$ promoter. Because of this promoter, expression of the cellular receptors only occurs in cells of the myeloid lineage. The receptor is part of the FAS apoptosis pathway, and normally binds to a ligand that induces apoptosis. The drug AP20187 causes a trimerization of these FAS receptors and leads to apoptosis through activation of the caspase 8 pathway (14). Enhanced green fluorescent protein (EGFP) was fused to the suicide gene to allow for easy identification of transgene-expressing cells. Some dendritic cells are also depleted upon addition of the drug AP20187; however, because of reduced expression of the MaFIA transgene, a smaller population of dendritic cells is depleted compared to macrophages. Neutrophils increase as a result of macrophage and dendritic cell depletion, but these cells are immature and their role is limited (15). The original MaFIA mice were created with wild-type C57Bl/6J mice purchased from Charles River Laboratories (Boston, MA, USA).

The object of the proposed research was to investigate the metastatic process in the presence and absence of macrophages. Since MaFIA mice can undergo the selective depletion of macrophages we examined the metastatic potential of tumors in the presence and absence of macrophages. With subdermal and intravenous injections of B16-F10 ATCC \# CRL-6475 melanoma cells, we compared induced metastasis from mice with depleted macrophages to control mice with macrophages. The subdermal injections were designed to create primary tumors to observe spontaneous metastasis. The intravenous injections were designed to simulate circulating tumor cells from the primary tumor, or experimental metastasis.

\section{Materials and methods}

Cell culture. A mouse melanoma cell line, B16 F10 ATCC \# CRL-6475, was grown in Dulbecco's modified Eagle's medium, (DMEM) supplemented with $10 \%$ bovine calf serum (Hyclone, Logan, UT, USA). Cells were kept in a $37^{\circ} \mathrm{C}$ incubator supplied with $5 \% \mathrm{CO}_{2}$ and sub-cultured every 2 days. To prepare cells for injection, the medium was changed $24 \mathrm{~h}$ before adding trypsin. Cells were trypsinized using $1 \%$ Trypsin (Gibco) dissolved in Hank's solution (Hyclone). A cell count was performed and cells were re-suspended at a concentration of $5 \times 10^{6}$ cells $/ \mathrm{ml}$.

Mice. Mice were cared for and used under proper Institutional Animal Care and Use Committee (IACUC) procedures and practices. Breeding pairs were established between two MaFIA positive mice. Not all mice were transgene positive, so pups were tested for transgene expression using a tail snip. Blood samples from the tail were examined using flow cytometry. Mice expressing the transgene also expressed GFP, while mice without the transgene did not. For the spontaneous and experimental metastasis experiments, mice were divided into two experimental groups. The control groups received injections of melanoma cells. The macrophage-depleted groups received depletion treatment and then the injection of melanoma cells. Subdermal and intravenous injections consisted of $1 \times 10^{6}$ cells in $200 \mu \mathrm{l}$ of growth medium. The mice were anesthetized using Avertin. Subdermal and intravenous injections were performed using a 27-gauge needle and a 1cc syringe. The subdermal injections were placed on the back of the mouse between the shoulders, while the intravenous injections were administered through tail veins.

Depletion. Depletion of the positive mice was achieved using the drug AP20187. The drug was dissolved in $100 \%$ ethanol at a concentration of $62.5 \mathrm{mg} / \mathrm{ml}$. Injection volumes were adjusted to administer $10 \mathrm{mg}$ of the drug AP20187 for every $\mathrm{kg}$ of mouse body weight. Drug formulation included sterile phosphate buffered saline (PBS), 2\% Tween in PBS, and polyethylene glycol (PEG) 400. Mice were weighed each day before injections. Intra-peritoneal injections were given using a 27-gauge needle and a 1cc syringe. Mice were given the fiveday depletion regimen as previously described (15). After the five-day period, mice were given injections three times a week to maintain depletion.

Tissue collection and staining. Peritoneal lavages were utilized to ensure that the depletion protocol functioned properly. A lavage was performed by filling the peritoneal cavity with $5 \mathrm{ml}$ of Hank's balanced salt solution (HBSS), injected with a 27-gauge needle and a 5-ml syringe. The cavity was then rubbed with a wet needle cap to shake the macrophages free from the tissues. The HBSS was then retrieved from the cavity using a 21-gauge needle and a 5-ml syringe. The cells were centrifuged at $650 \mathrm{x}$ g for $5 \mathrm{~min}$ and then re-suspended in $500 \mu \mathrm{l}$ of HBSS and analyzed using flow cytometry for the quantification of GFP-positive cells.

The tumors, from subdermal and intravenous injections, were allowed to grow for two weeks after which the mice were sacrificed by $\mathrm{CO}_{2}$ asphyxiation. The lungs, kidneys and tumors 
A

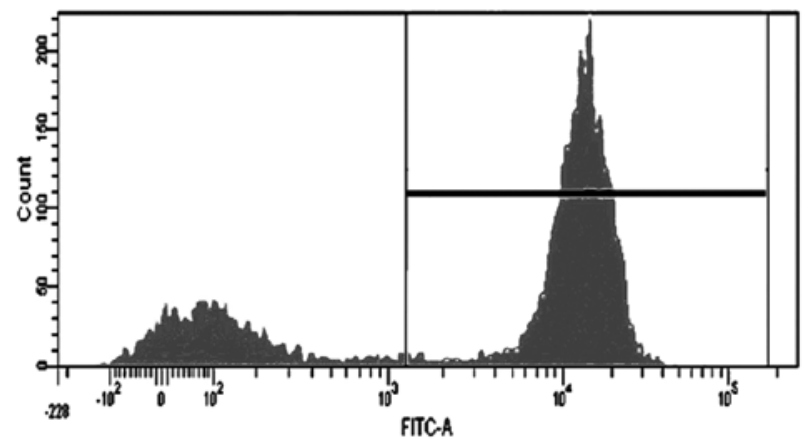

B

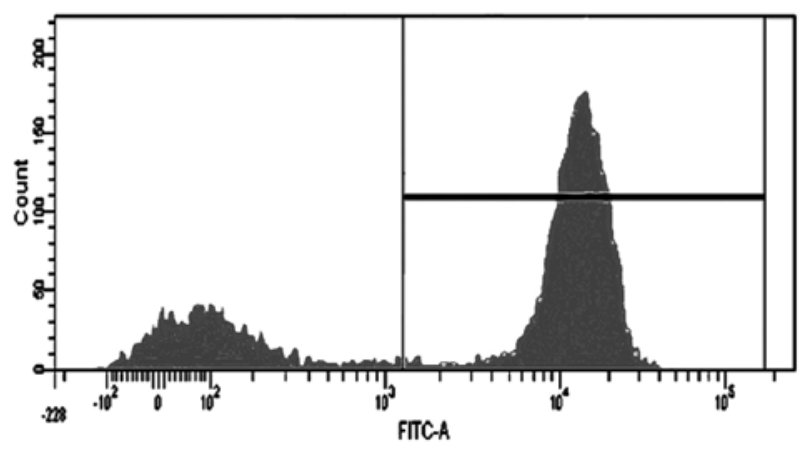

C

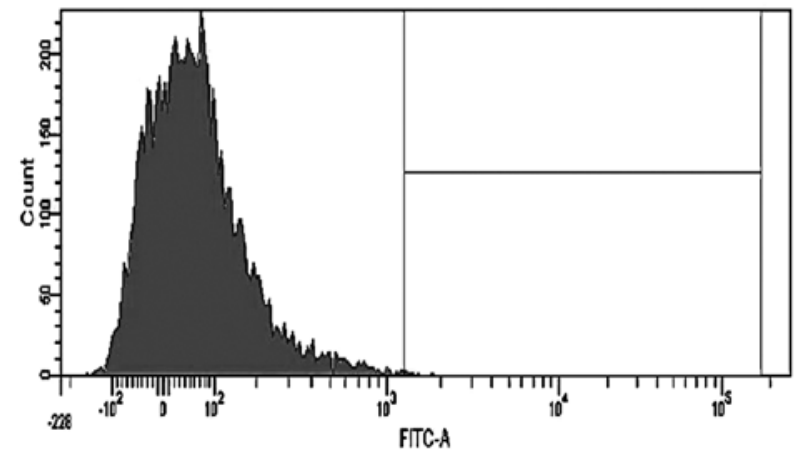

Figure 1. Depletion results, flow cytometry results from peritoneal lavages of depletion studies in mice. Each histogram represents the amount of GFP expressed. These histograms are representative of all of the mice used to test for the depletion protocol. (A) Untreated control group, (B) mock-treated group and (C) treated group.

of each mouse were removed for analysis of melanoma cell content. The organs were placed into a 24-well plate, and each well was filled with $1.5 \mathrm{ml}$ of HBSS. The organs were then macerated with scissors and transferred into a stomacher bag filled with $1.5 \mathrm{ml}$ of HBSS to be homogenized. Lungs were incubated with $15 \mu \mathrm{l}$ of DNASE collagenase solution at $37^{\circ} \mathrm{C}$ for $1 \mathrm{~h}$. Once the organs were homogenized, the sample was divided in half and filtered. The samples were centrifuged at $900 \times \mathrm{g}$ for $5 \mathrm{~min}$ and the supernatant was decanted. A red blood cell lysis was performed by adding $2 \mathrm{ml}$ of Tris ammonium chloride lysis buffer, $\mathrm{pH} 7.5$ at $50 \mathrm{nM}$, and incubating for 2-5 min. After $5 \mathrm{~min}, 2 \mathrm{ml}$ of HBSS was added and samples were again centrifuged and the supernatant decanted. An Fc block, (Mouse BD Fc Block ${ }^{\mathrm{TM}}$ ), was added to samples containing macrophages and allowed to incubate for $10 \mathrm{~min}$ on ice and then the samples were washed twice with HBSS. An aliquot of $50 \mu \mathrm{l}$ of a 1:100 dilution of the combined melanoma antibodies HMB45ab732, DT101ab732 and BC199ab732 (Abcam Inc., Cambridge, MA, USA) was added. These antibodies are specific for melanoma cells. The antibodies were allowed to incubate for $1 \mathrm{~h}$ on ice in the dark. The samples were then washed twice with HBSS and stained with a secondary antibody conjugated with Alexa Fluor 633, on ice for $1 \mathrm{~h}$. The samples were washed twice and then analyzed using flow cytometry.

Flow cytometry. Flow cytometric analysis was performed on a BD FACSCanto. Cell sorting was performed on the FACSVantage machine. Cells were sorted into tubes containing $1 \mathrm{ml}$ of bovine calf serum. Data were collected and analyzed using FACSDIVA software.

Slide preparation and analysis. Samples from the cell sort and organ samples were placed onto slides using a Cytospin. The slides were stained using May Grunwald stain, 4\% Geimsa, and then rinsed briefly in distilled water. Some slides were not stained with the May Grunwald and Geimsa, but analyzed for fluorescence. A cover slip was placed on top of each slide with a drop of Cytoseal. Slides were analyzed using a Zeiss Axioscop fluorescent microscope.

\section{Results}

Initially macrophage depletion was confirmed by conducting a depletion study with three cohorts (10 mice each) of MaFIA positive mice. One cohort designated as a control was left untreated, another was mock-treated using vehicle only, and the third was treated with AP20187. Mice were injected for five consecutive days as previously described (15) and then sacrificed $24 \mathrm{~h}$ following the last injection. Peritoneal lavages were performed on all three cohorts of mice and analyzed by flow cytometry (a representative graph of one mouse from each cohort is shown in Fig. 1). Once inserted, the transgene expression of GFP could be visualized in the macrophages using a fluorescent microscope. The macrophages from AP20187-treated mice showed a GFP level of 2\%, mocktreated mice showed $61 \%$, and the positive control showed $73 \%$, indicating that the depletion protocol was effective. Lungs and kidneys were also tested and showed a similar trend (data not shown).

Spontaneous metastasis. Subdermal injections were administered and the lungs and kidneys were harvested and analyzed for migrated melanoma cells. Samples were stained with a cocktail of melanoma specific antibodies (namely HMB45ab732, DT101ab732 and BC199ab732) (Abcam Inc.), and then a secondary antibody, conjugated with Alexa Fluor 633. Flow cytometry analysis from the control group of the harvested lungs showed that $55 \%(\mathrm{~N}=20, \mathrm{P}=0.008)$ of the harvested cells stained positive, while the lung samples from the macrophagedepleted mice showed only $6 \%(\mathrm{~N}=20, \mathrm{P}=0.008)$ of the cells stained positive or the melanoma markers (Fig. 2).

Flow cytometry analysis of the samples from the kidney control group showed that $55 \%(\mathrm{~N}=20, \mathrm{P}=0.014)$ of the 
A

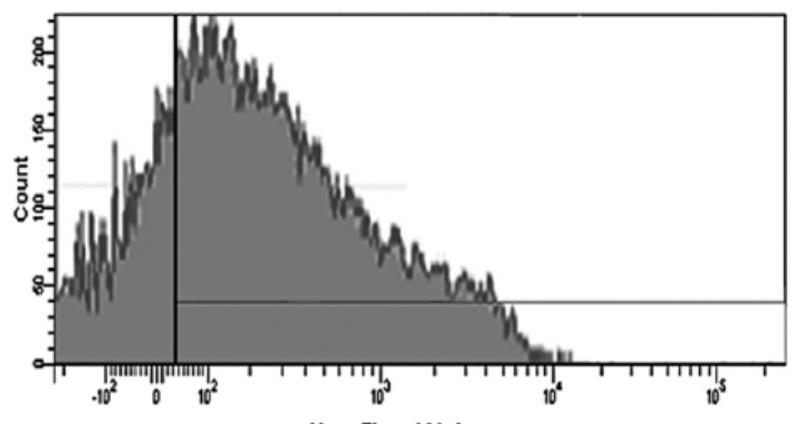

Alexa Fluor $663-\mathrm{A}$

B

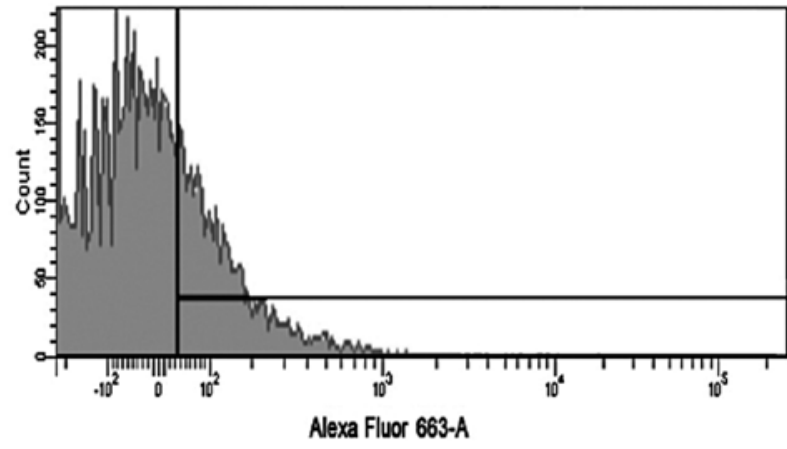

C Spontaneous metastasis lung $(p=0.008)$

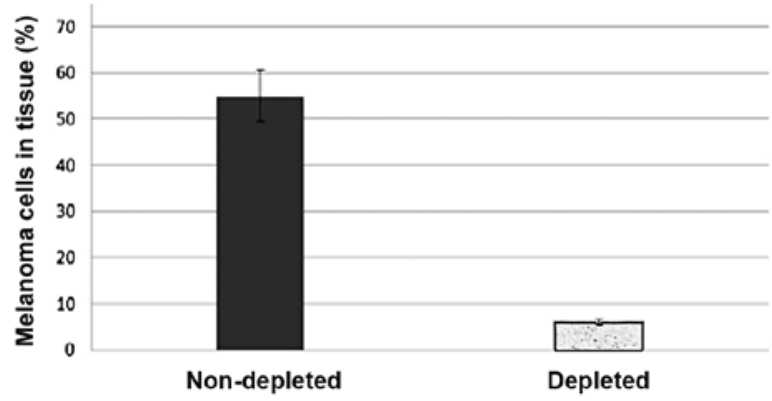

Figure 2. Spontaneous metastasis to the lungs following macrophage depletion, (A and B) are flow cytometry results from the harvested lung tissues. The histograms represent the amount of Alexa Fluor 633 detected. (A) The tumor control (with macrophages), and (B) the macrophage-depleted group. (C) The findings in bar graph form. A total of 20 mice were tested. Error bars are \pm one standard deviation all of the mice used to test for the depletion protocol. (A) The untreated control group, (B) mock-treated group and (C) the treated group.

harvested cells stained positive, while samples from the depleted mice showed $23 \%(\mathrm{~N}=20, \mathrm{P}=0.014)$ of the cells stained positive (Fig. 3).

Experimental metastasis. Intravenous injections of melanoma cells through the tail vein of the mice were also administered and the lungs and kidneys were harvested and analyzed in the same manner as the subdermal injected mice. Experimental metastasis flow cytometry data from lung control samples showed that $3 \%(\mathrm{~N}=8, \mathrm{P}=0.001)$ of the harvested cells stained positive with the anti-melanoma antibodies. The lung samples from the depleted mice showed that $19 \%(\mathrm{~N}=8, \mathrm{P}=0.001)$ of the cells stained positive for the melanoma markers (Fig. 4).

\section{A}

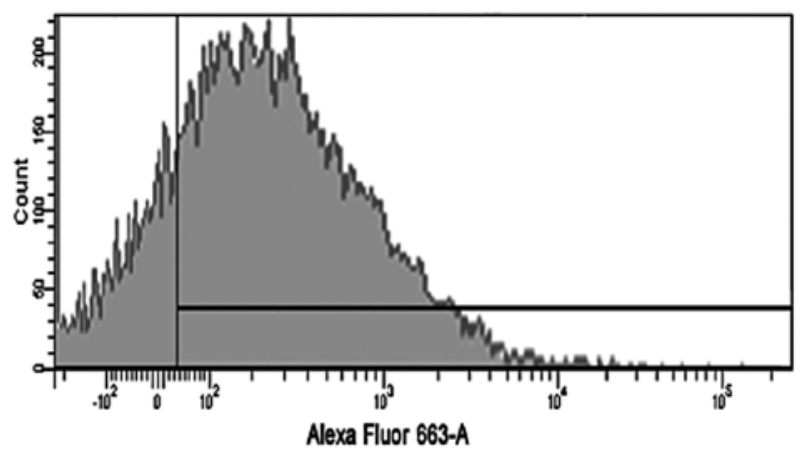

B

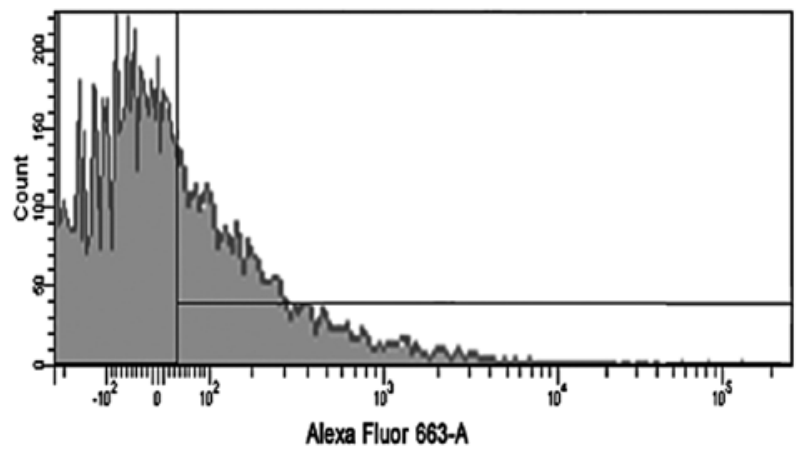

C

Spontaneous metastasis

kidney $(p=0.014)$

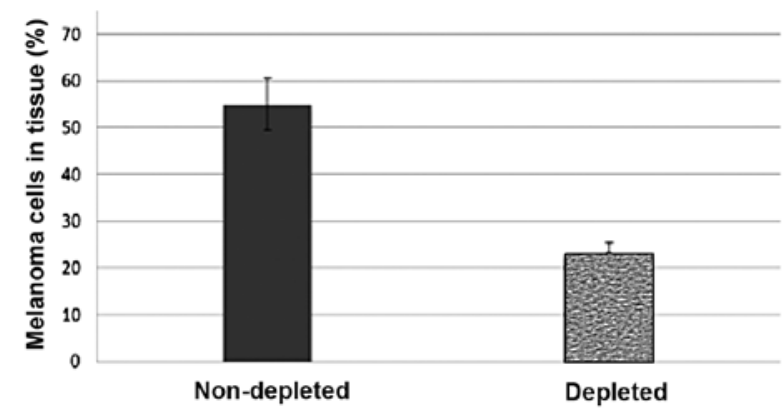

Figure 3. Spontaneous metastasis to the kidneys following depletion, (A and B) are flow cytometry results from the harvested kidney tissues. The histograms represent the amount of Alexa Fluor 633 detected. (A) The tumor control (with macrophages), and (B) is the macrophage-depleted group. (C) The findings in bar graph form. A total of 20 mice were tested. Error bars are \pm one standard deviation.

Flow cytometry data from kidney control samples showed that $2.5 \%,(\mathrm{~N}=8, \mathrm{P}>0.01)$, of the harvested cells stained positive with the melanoma markers. The kidney samples from the depleted mice showed that $8 \%(\mathrm{~N}=8, \mathrm{P}>0.01)$ of the cells stained positive for the melanoma markers (Fig. 5).

Double-positive cells. From the spontaneous metastasis experiment, we observed double-positive cells in the tumors, lungs and kidneys of the non-depleted mice. These doublepositive cells expressed both GFP and stained positive for the anti-melanoma antibodies. This was a possible indication of macrophage/melanoma fusion. By means of both immunohistochemistry and immunofluorescence we further examined the double-positive cells. We exposed macrophages taken 
A

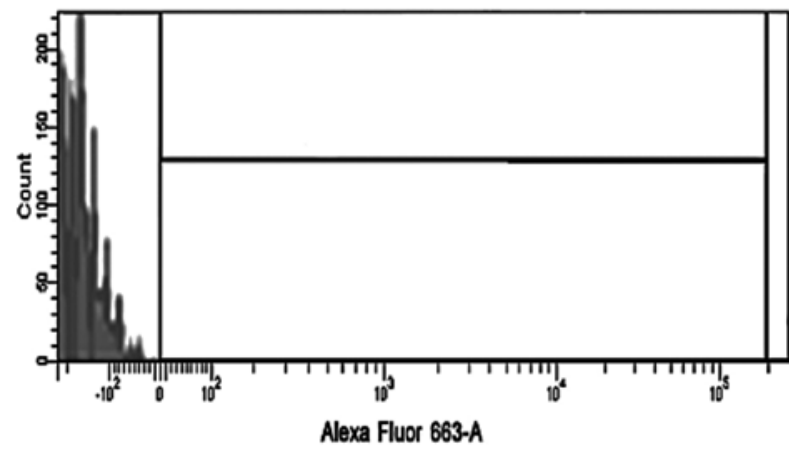

B

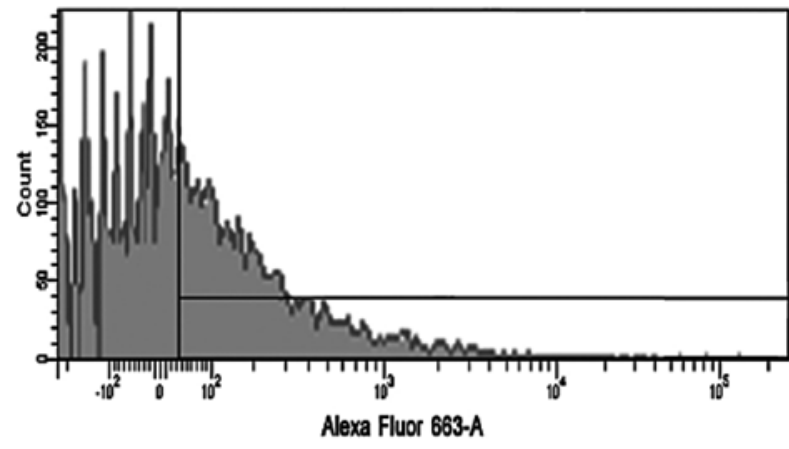

C

Experimental metastasis lung $(p=0.001)$

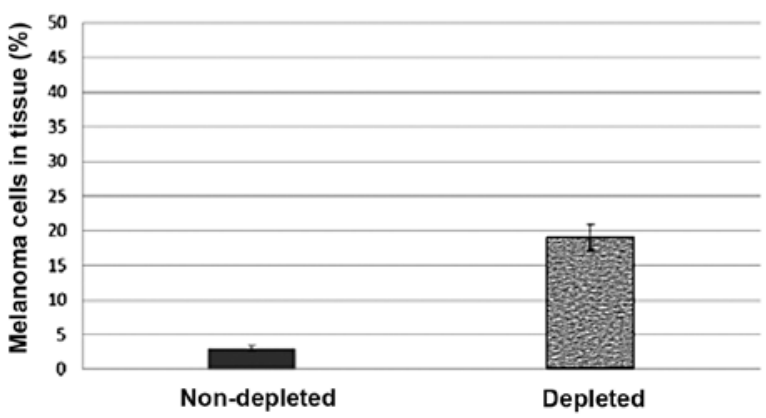

Figure 4. Experimental metastasis to the lungs following depletion, (A and B) are flow cytometry results from the harvested lung tissues. The histograms represent the amount of Alexa Fluor 633 detected. (A) The tumor control, (with macrophages), and (B) the depleted group. (C) The findings in bar graph form. A total of 8 mice were tested. Error bars are \pm one standard deviation.

from a lavage of a MaFIA mouse to melanoma cell debris to see if the macrophage MHC presentation yields false doublepositives. Based on the results (Figs. 6 and 7), it was clear that MHC presentation did not yield any double-positive cells.

\section{Discussion}

Many sources indicate that the presence of TAMs increases the metastatic potential of cancerous cells, or that TAMs are directly involved in metastasis (8-13). Our preliminary data are supportive evidence that TAMs play a role in facilitating metastasis. In mice that received subdermal injections, we noted a reduction in migrated melanoma cells when the macrophages were selectively depleted. This suggests that
A
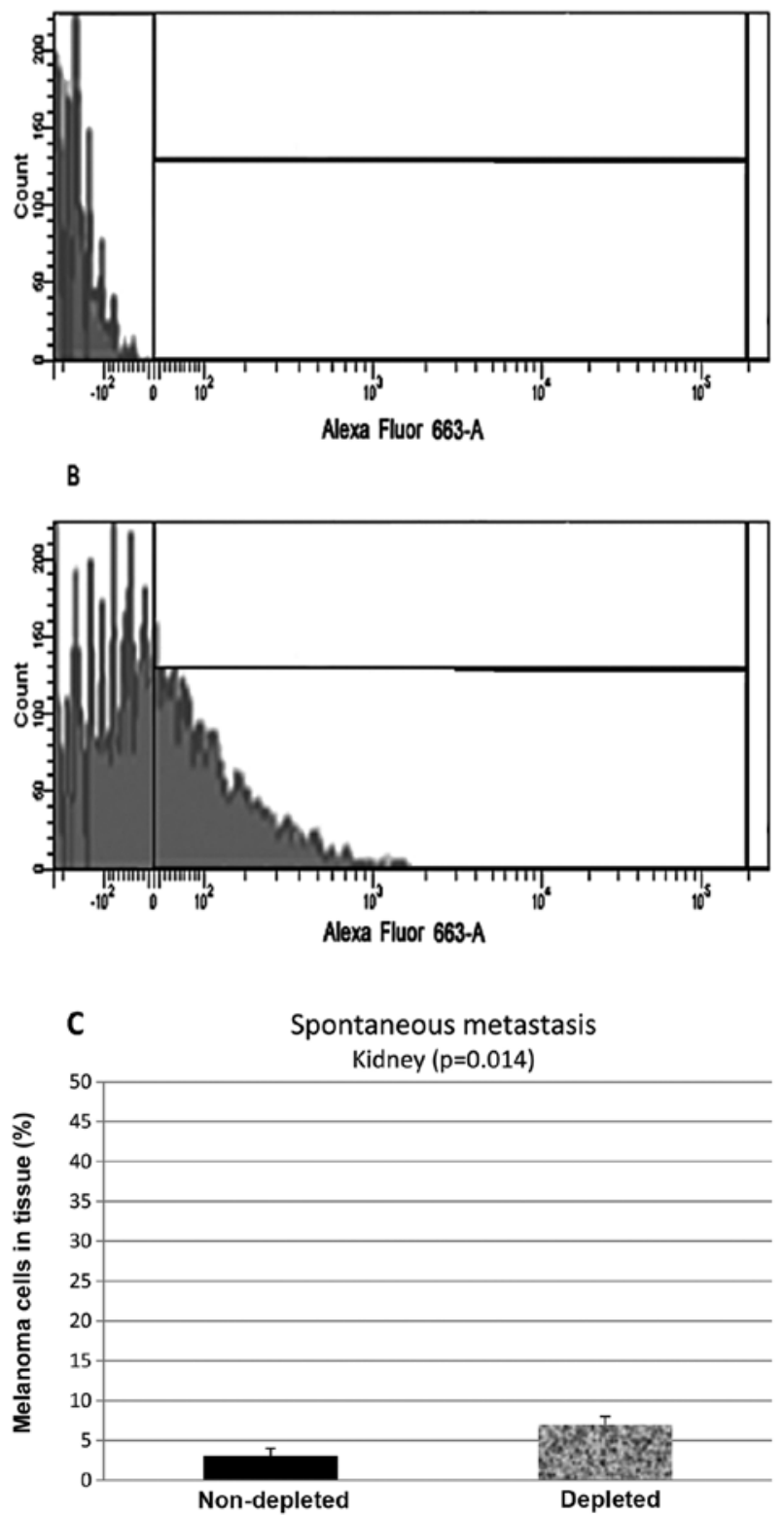

Figure 5. Experimental metastasis to the kidneys following depletion, (A and B) are flow cytometry results from the harvested kidney tissues. The histograms represent the amount of Alexa Fluor 633 detected. (A) The tumor control (with macrophages), and (B) the macrophage-depleted group. (C) The findings in bar graph form. A total of 8 mice were tested. Error bars are \pm one standard deviation.

macrophages facilitate the migration of melanoma cells from the primary tumor site to the lungs and kidneys. There was a significant difference between the control (untreated group) and the macrophage-depleted group with regards to the number of melanoma cells found in both the lungs $(\mathrm{P}=0.008)$ and the kidneys $(\mathrm{P}=0.014)$.

The results from the experimental metastasis experiment indicated that a decrease in macrophages enables melanoma cells, already in the blood stream, to have a greater metastatic potential. The lungs in macrophage-depleted mice had a significantly increased level of metastasis when compared to the control (untreated) mice. From the results obtained, it could be suggested that the presence of macrophages in the 
A

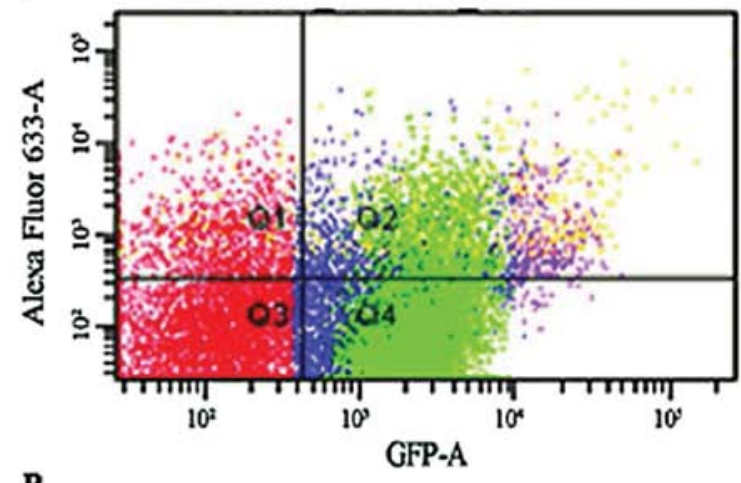

B

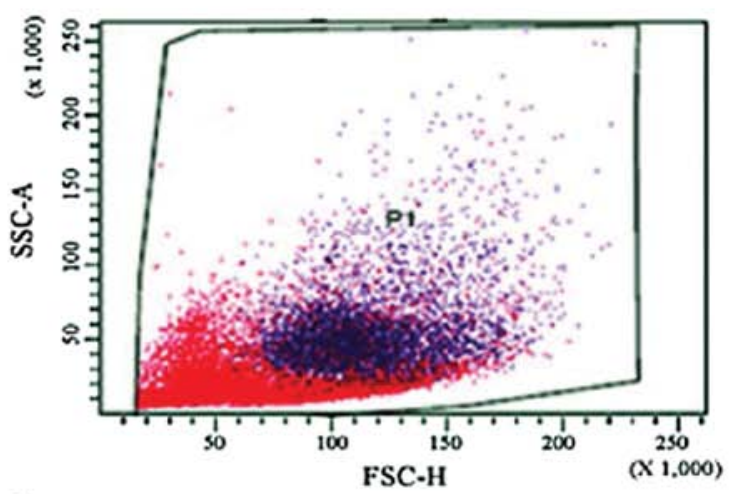

C

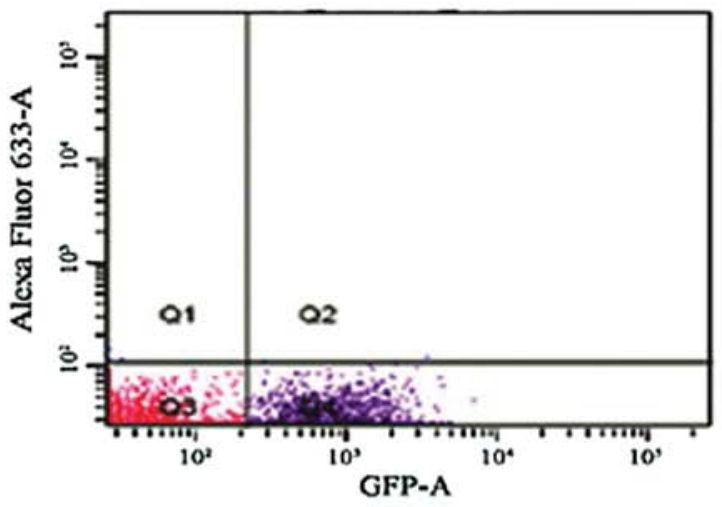

Figure 6. Graphs of double-positive cells. (A) flow cytometry data for the double-positive cells. The Q2 region shows the double-positive cells that express GFP, along the $\mathrm{x}$-axis, and Alexa Fluor 633 along the $y$-axis. (B and C) Flow cytometry data. (B) Population of Live cells exposed to melanoma debris. (C) No double-positive cells were seen when Macrophages were exposed to melanoma debris.

lungs helps to prevent metastatic spread. The same trend was also seen in the experimentally-induced metastases in the kidneys. There was also a significant difference in the amount of metastasis in the kidneys when comparing the control and experimental groups, suggesting that the kidney metastasis is also influenced by the presence of macrophages in the kidneys.

Since dendritic cells were also depleted it is possible that they are also involved in metastasis. The likelihood of their involvement is minimal because they have a reduced expression of the MaFIA transgene, and therefore a smaller population of dendritic cells is depleted compared to macrophages. Second, the depletion of dendritic cells is delayed. Seven days after the 5 day depletion regimen, the percentage of depleted cells increased from 48 to $98 \%$. Since mice were sacrificed 2
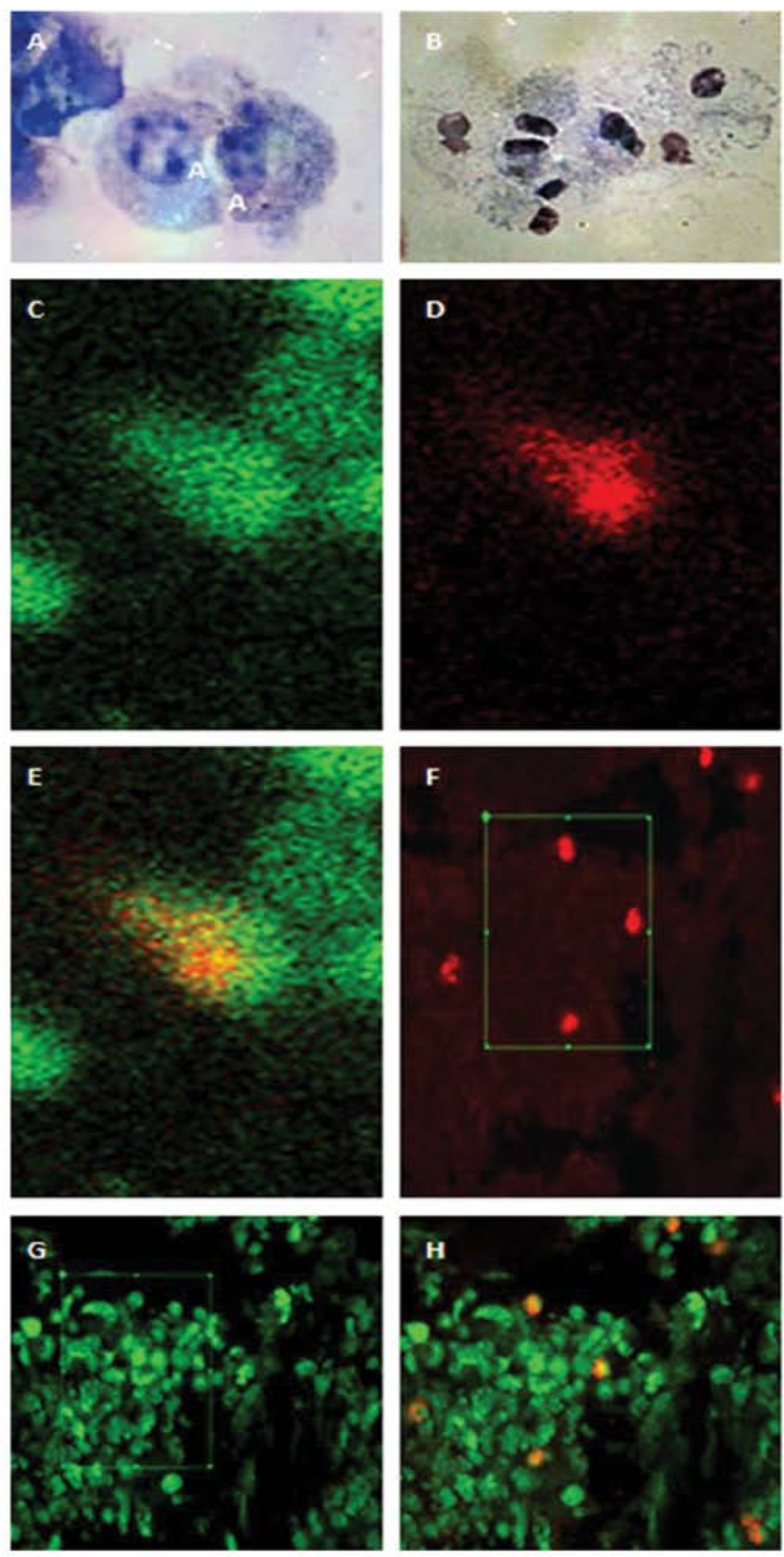

Figure 7. Micrographs of Giemsa stains of tumor double-positive cells, (A and B) are Giemsa stains of tumor and lung double-positive cells, respectively, magnified to x100. (D-F) Confocal microscopy images of the same macrophages. (D and E) GFP and Alexa Fluor expression, respectively. (F) Using both fluorescents some double-positive cells are seen expressing both GFP and Alexa Fluor. ( $\mathrm{G}$ and $\mathrm{H}$ ) A cross section a tumor from control MaFIA mouse using both fluorescents. (G and H) Alexa Fluor 633 and GFP, respectively.

weeks after the 5 day depletion regimen, the effects of reduced dendritic cells would be reduced compared to macrophages. This leads us to believe that the mice were sacrificed before any significant effects from the loss of dendritic cells occurred. Neutrophils increase as a result of macrophage and dendritic cell depletion, and it is also possible that their increase could be involved in the decrease in metastasis. The likelihood of this occurring is limited since the neutrophils produced in response to deficient macrophages and dendritic cells are immature $(14,15)$. 
By comparing both the spontaneous and experimental metastasis systems, we can see further evidence for TAM involvement in metastasis. The data are suggestive of TAMs serving as an aid to cancer metastasis. The involvement of TAMs in metastasis is further supported by the number of double-positive cells (Fig. 7). These are cells that stain positive for melanoma markers and are also positive for GFP expression. GFP is expressed only in the myeloid cell line; therefore the double-positive cells indicate possible cell fusion. Since EGFP was placed in the suicide gene of transgene-expressing cells, macrophages express GFP. Fig. 7D-F indicate that cells that migrated from target organs can express both GFP and melanoma surface markers as indicated by the binding of the anti-melanoma antibodies, which leads us to believe that this is evidence of TAM/melanoma fusion. It is possible that the tumors convert naturally programmed M1 macrophages into type M2 cells. These M2 TAMs can then be utilized as carrier cells which facilitate metastasis. The disruption of a tumor's ability to convert macrophages from M1 to M2, or inhibiting macrophages from fusing with cancerous cells could be potential targets for cancer therapies. Further research needs to be conducted to confirm that macrophages facilitate metastasis and that tumors provide the means for the conversion of macrophage phenotype.

\section{Acknowledgements}

Funding was provided by a Mentoring Environment Grant (MEG) from Brigham Young University. MaFIA mice were provided by Dr Sandra Burnett, originally developed by the University of Kentucky, Division of Laboratory Animal Resources (Lexington, KY, USA). A. Clement and K. Wells are also acknowledged for their help with the mouse procedures.

\section{References}

1. Mantovani A, Sozzani S, Locati M, Allavena P and Sica A: Macrophage polarization: tumor-associated macrophages as a paradigm for polarized M2 mononuclear phagocytes. Trends Immunol 11: 549-555, 2002.

2. Eubank TD, Galloway M, Montague CM, Waldman WJ and Marsh CB: M-CSF induces vascular endothelial growth factor production and angiogenic activity from human monocytes. J Immunol 171: 2637-2643, 2003.
3. Elgert KD, Alleva DG and Mullins DW: Tumor-induced immune dysfunction: the macrophage connection. J Leukocyte Biol 64: 275-290, 1998.

4. Lewis CE and Pollard JW: Distinct role of macrophages in different tumor microenvironments. Cancer Res 66: 605-612, 2006.

5. Nardin A and Abastado JP: Macrophages and cancer. Front Biosci 13: 3494-3505, 2008.

6. Lewis C and Murdoch C: Macrophages responses to hypoxia: implications for tumor progression and anti-cancer therapies. Am J Pathol 167: 627-635, 2005.

7. Polverini PJ and Leibovich SJ: Effect of macrophage depletion on growth and neovascularization of hamster buccal pouch carcinomas. J Oral Pathol 16: 436-444, 1987.

8. Yamaguchi $\mathrm{H}$, Wyckoff $\mathbf{J}$ and Condeelis $\mathrm{J}$ : Cell migration in tumors. Curr Opin Cell Biol 17: 559-564, 2005.

9. Rachkovsky M, Sodi S, Chakraborty A, Avissar Y, Bolognia J, McNiff JM, Platt J, Bermudes D and Pawelek J: Melanoma X macrophage hybrids with enhanced metastatic potential. Clin Exp Metastasis 16: 299-312, 1998.

10. Hagemann T, Robinson SC, Schulz M, Trümper L, Balkwill FR and Binder C: Enhanced invasiveness of breast cancer cell lines upon co-cultivation with macrophages is due to TNF-alpha dependent up-regulation of matrix metalloproteases. Carcinogenesis 25: 1543-1549, 2004.

11. Goswami S, Sahai E, Wyckoff JB, Cammer M, Cox D, Pixley FJ, Stanley ER, Segall JE and Condeelis JS: Macrophages promote the invasion of breast carcinoma cells via a colony-stimulating factor-1/epidermal growth factor paracrine loop. Cancer Res 65: 5278-5283, 2005.

12. Wyckoff J, Wang W, Lin EY, Wang Y, Pixley F, Stanley ER, Graf T, Pollard JW, Segall J and Condeelis J: A paracrine loop between tumor cells and macrophages is required for tumor cell migration in mammary tumors. Cancer Res 64: 7022-7029, 2004.

13. Pollard JW: Tumor-educated macrophages promote tumor progression and metastasis. Nat Rev Cancer 4: 71-78, 2004.

14. Burnett SH, Kershen EJ, Zhang J, Zeng L, Straley SC, Kaplan AM and Cohen DA: Conditional macrophage ablation in transgenic mice expressing a Fas-based suicide gene. J Leukoc Biol 75: 612-623, 2004

15. Allende ML, Bektas M, Lee BG, Bonifacino E, Kang J, Tuymetova G, Chen W, Saba JD and Proia RL: Sphingosine1-phosphate lyase deficiency produces a pro-inflammatory response while impairing neutrophil trafficking. J Biol Chem 286: 7348-7358, 2011. 